\section{Análisis del crecimiento del desempleo en el Ecuador: período 2010-2021}

Analysis of unemployment growth in Ecuador: period 2010-2021

\section{RESUMEN}

El desempleo es una variable de especial atención en la economía ecuatoriana, por ello conocer su evolución es primordial para mejorar las condiciones de vida de la población y de la economía en general. Un país con una elevada tasa de desempleo desencadena factores negativos tales como: pobreza, informalidad, delincuencia, migración, etc. Por lo tanto, este trabajo tiene como objetivo analizar el crecimiento del desempleo en el Ecuador durante el periodo 2010-2021. Para ello se realiza una investigación de tipo analítica-histórica-descriptiva, sustentando la información de varias fuentes oficiales nacionales. Los resultados reflejan que el crecimiento del desempleo en el país es más evidente en la zona urbana y en la actividad laboral femenina. Para el 2018 ya se reflejaba la caída del empleo formal debido a la crisis derivada del 2014, lo cual daba paso al incremento del empleo informal o en últimas estancias al desempleo, situación que se incrementó como efecto de la pandemia pero que, en comparación a los demás países de la región, se ubicó en índices aceptables a pesar de la crisis social y económica experimentada. Además, el aumento de los impuestos, la apreciación del dólar, menor demanda interna, daños causados por el sismo son algunas causas con alta relevancia en el incremento del desempleo.

Palabras clave: Desempleo; economía; migración; pandemia; pobreza

\section{ABSTRACT}

Unemployment is a variable of special attention in the Ecuadorian economy, therefore knowing its evolution is essential to improve the living conditions of the population and the economy in general. A country with a high unemployment rate triggers negative factors such as: poverty, informality, crime, migration, etc. Therefore, this work aims to analyze the growth of unemployment in Ecuador during the period 2010-2021. For this, analytical-historical-descriptive research is carried out, supporting information from various official national sources. The results reflect that the growth of unemployment in the country is more evident in urban areas and in female labor activity. By 2018, the fall in formal employment was already reflected due to the crisis derived from 2014, which gave way to the increase in informal employment or in the last few stays to unemployment, influencing to a greater extent in 2020 due to the COVID-19 pandemic. 19. In addition, the increase in taxes, the appreciation of the dollar, lower domestic demand, damage caused by the earthquake are some causes with high relevance in the increase in unemployment.

Keywords: Unemployment; economy; migration; pandemic; poverty
RELIGACIÓN

REVISTA DE CIENCIAS SOCIALES Y HUMANIDADES JOURNAL OF SOCIAL SCIENCES AND HUMANITIE
REVISTA DE CENCIAS SOCIAIS E HUMANAS

\section{INFORMACIÓN:}

http://doi.org/10.46652/rgn.v6i30.850 ISSN $2477-9083$

Vol. 6 No. 30, 2021. e210850 Quito, Ecuador

Enviado: octubre 12, 2021

Aceptado: diciembre 10, 2021

Publicado: diciembre 18, 2021

Publicación Continua

Sección General | Peer Reviewed
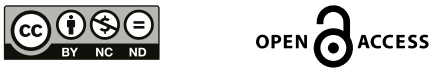

AUTOR:

(D) Josthin Ramirez

Universidad Técnica de Machala -

Ecuador

jramirez5@utmachala.edu.ec

(D) Jonh Campuzano

Universidad Técnica de Machala Ecuador

jcampuzanov@gmail.com

Conflicto de intereses

Los autores declaran que no existe conflicto de interés posible.

Financiamiento

No existió asistencia financiera de partes externas al presente artículo.

Agradecimiento

Quiero dar gracias a Dios y a mi mamá por ser fuente de inspiración y apoyo incondicional para cumplir mis metas.

Nota

N/A
ENTIDAD EDITORA 


\section{Introducción}

A lo largo de la historia el desempleo ha sido considerado como un problema social para las economías. Desde el término genérico, el desempleo hace referencia a un estado de ocupación de los individuos, en el cual, aquellas personas que desean trabajar no encuentran un empleo. Considerando este contexto para Chen (1990) el desempleo tiene connotaciones específicas de precios ni de condiciones de aceptación, siendo el trabajo el medio que permite que las personas puedan asegurarse un determinado nivel de vida, para sí mismas y para sus familias.

Los problemas relacionados con el trabajo, el empleo o el desempleo y su impacto en el bienestar social son el eje central del análisis económico. Sin embargo, en diferentes métodos teóricos, su tratamiento y atención son sumamente diversos, sus conclusiones también son muy diferentes, y en la mayoría de los casos, las recomendaciones normativas derivadas de ellos también son muy contradictorias. De esta manera es preciso acotar que, desde el origen de la Economía como ciencia, esta se encuentra relacionada con el desarrollo económico de una nueva forma de organización social, que llegó a ser conocida como el capitalismo que de acuerdo con Polany (1994) se refería principalmente a la generalización de los mercados y la conversión de todos los recursos productivos en mercancías.

De esta manera hicieron su aparición los mercados de recursos como el de capital, la tierra o el trabajo, lo cual implicaba que también el trabajo humano, bajo el régimen asalariado sobre la que se sustentaba la relación laboral existente en el mercado, llegaba también a convertirse en una mercancía más. El resultado lógico es que los economistas en el principio querían conocer la naturaleza económica de esta nueva mercancía, su valor y precio, el entorno en el que se usaba más o menos y qué podía promover mejor su uso para crear riqueza. Si bien varios de estos estudiosos, entre los que destacaban nombres como Cantillon, Locke o Condillac ya discutían sobre la naturaleza económica del trabajo en realidad fue Adam Smith quien primero se refirió a él para vincularlo al valor que da origen a cualquier forma de riqueza.

El trabajo anual de cada nación es el fondo que en principio la provee de todas las cosas necesarias y convenientes para la vida, y que anualmente consume el país. Dicho fondo se integra siempre, o con el producto inmediato del trabajo, o con lo que mediante dicho producto se compra de otras naciones (Smith, 1958, p. 53)

Al considerar el intercambio de mercado como el eje principal de la actividad económica, es necesario descubrir las causas fundamentales de la relación de cambio y las circunstancias que pueden conducir a la evolución aparentemente inestable de los precios de las mercancías. La respuesta se encuentra en un concepto básico, el valor, según Adam Smith, su origen y medida están en el trabajo. Smith indicaba definición de empleo o pleno empleo está determinado por los cambios en los salarios, tratando de decir: cuando los salarios son altos, se producirá el desempleo, y cuando los salarios (bajos) cambian, el pleno empleo seguirá existiendo porque aumenta la población ocupada.

Por otro lado, la teoría keynesiana se refiere al desempleo como aquella situación en la que el número de personas que se encuentra en la búsqueda de trabajo sobrepasa el número de puestos de trabajo que están disponibles y también al número de puestos de trabajo remunerados 
con los salarios actuales, siendo su explicación principalmente la falta de demanda efectiva de los bienes y servicios. Desde esta perspectiva, el análisis del empleo está contenido en las condiciones generales del proceso de acumulación, especialmente determinado por la evolución de los factores que inciden en la productividad, la misma que se encuentra determinada por un número determinado de factores, donde se encuentra el empleo. Los capitalistas pueden usar mano de obra. Por lo tanto, los conflictos laborales formados en la búsqueda de recursos que pueden afectar estas condiciones, o profundizarán la explotación, o lograrán la erradicación o reducción de la explotación (Torres \& Montero, 2005).

Actualmente, en sus concepciones más modernas, la Organización Internacional del Trabajo (2014) definió al desempleo como:

\begin{abstract}
El desempleo es una situación que se da cuando la cantidad de personas que buscan trabajo (demanda de empleo) excede el número de empleos disponibles (oferta de empleo). En los países en desarrollo, el desempleo es un concepto que se refiere principalmente al mercado de trabajo formal, el cual suele ser más pequeño que el informal, incluso a veces de manera considerable (Organización Internacional del Trabajo, 2014).
\end{abstract}

En este sentido, y considerando la importancia de este, la creación de fuentes de empleo y al mismo tiempo la reducción de la tasa de desempleo son objetivos que comparten todos los países. En América Latina y el Caribe, sigue siendo muy discutida la causa del desempleo y como contribuir en el crecimiento económico de la población, de esta manera se encuentra muy presente el interés de conocer las características que tiene el mercado laboral (López, 2020). No cabe duda de que existe un gran interés por el desempleo debido a que es una de las variables macroeconómicas de mayor importancia para el diseño de políticas públicas. Estadísticamente, la Organización Mundial del Trabajo demostró que la tasa de desocupación en el año 2020 fue del $11,1 \%$, y cerca de 28 millones de personas que trabajan no ganan lo suficiente para mantenerse junto a sus familias por encima de la línea de la pobreza.

En América Latina las tasas de desempleo se han ido incrementando paulatinamente en los últimos años, es así como 34 millones de personas se encuentran buscando un puesto de trabajo sin conseguirlo. Como se pueden inferir a partir de los datos mencionados, la situación en América Latina se presenta preocupante, es así que, considerando la importancia del tema, en la presente investigación se analiza el crecimiento del desempleo en el Ecuador en el periodo 2010 al 2021.

Poniendo en contexto la situación del desempleo en Ecuador, después del año 2000, el país se enfrentaba a una gran crisis económica, en promedio la tasa de desempleo se ubicaba alrededor del 12\%, situación que se mantuvo constante hasta aproximadamente el 2009. En el año 2010 la tasa de desempleo se había reducido hasta ubicarse en el 5\% y en el año 2018 su punto máximo fue de $3.7 \%$, lo cual fue resultado de la implementación de programas para la creación de empleo como las obras en construcción, proyecto de innovación hidráulica, construcción de vías, entre otras, fueron parte de los mecanismos para el descenso del indicador de pobreza y desempleo (León et al., 2020). 
la importancia de que las tasas de desempleo hubiesen disminuido se explica por el hecho de que tanto en el sector económico como social es importante conocer que "Los altos índices de desempleo y de informalidad contribuyen a explicar la pobreza y la extrema desigualdad imperantes en el país" (Vallejo, 2010, p. 8). La lucha contra el desempleo es clave a favor del desarrollo económico de un país, sin embargo; "El crecimiento económico por sí solo no se traduce necesariamente en una mayor cantidad y una mejor calidad de empleos, especialmente para los sectores más pobres, vulnerables y aquellos en riesgo de quedar marginados" (OIT, 2021, p. 1).

Aunque el efecto del desempleo afecta a toda la población, autores como Rodríguez (2018) han identificado que las mujeres y el sector más joven de la población son los que se ven mayormente afectados, situación que se puede demostrar con los datos del año 2016, donde las mujeres registraron un incremento de 2 puntos porcentuales (9,8\%) con respecto al 2015 (7,8\%), y el desempleo en los jóvenes incremento más de 3p.p. (18,3\%) con respecto al 2015 (15,1\%). Los números en cuanto al empleo informal tampoco se mostraban alentadores debido a que, si bien las tasas de desempleo no se incrementaban de forma drástica, se observaba un hecho contradictorio cuando este tipo de empleo se venía incrementando desde el 2015, haciendo que para el año 2016 se registrasen 134 millones de personas con un empleo de mala calidad. Con respecto a los salarios reales, los mencionados autores, indican que en el año 2015 se registró una disminución del -1,3\%, para los años posteriores esta situación se acentúo en mayor medida.

El problema del desempleo en la sociedad moderna se ve agravado por las continuas crisis económicas, políticas, sociales y ambientales, así como por el progreso de la era en la que las nuevas tecnologías marcan el desarrollo de la productividad y, por lo tanto, cambian el factor trabajo (Briales, 2017). Con la llegada del COVID-19 el mercado de trabajo sufrió un grave impacto. Sin duda la situación del desempleo es desfavorable para la economía ecuatoriana, que lamentablemente se ha visto incrementada desde el año 2020 con la pandemia provocada por el COVID-19 y las medidas de confinamiento tomadas por el gobierno. De acuerdo con la Comisión Económica para América Latina y el Caribe (2021), la crisis que surgió como consecuencia del contagio del virus tiene un gran impacto en la economía y en los mercados laborales. Para finales del 2020 el PIB se ubicó en el 7.1\% lo cual se pudo analizar en la pérdida de empleos y un aumento de la tasa de desocupación, que alcanzó el 10,5\%.

Gran parte del deterioro del mercado laboral rural es consecuencia de la pandemia de COVID-19. Entre el 16 de marzo de 2020 y el 4 de marzo de 2021 en los sectores de agricultura, ganadería, silvicultura y pesca se perdieron 30.418 empleos adecuados, según el Ministerio de Trabajo (Primicias, 2021). Pero también ya se empieza a notar cierta reactivación, de acuerdo con los análisis de la Comisión Económica para América Latina y el Caribe (2021), en la medida en que las restricciones se levantan se observa como paulatinamente la población retoma sus actividades productivas y por lo tanto se espera una lenta recuperación del empleo, aunque no cabe duda de que durante el 2021 la tasa de desocupación y la precariedad laboral serán una constante.

En consecuencia, la recuperación del mercado laboral y la economía de América Latina y El Caribe serán mucho más lento (Comisión Económica para América Latina y el Caribe, 2020). Además, la migración de un país cercano como Venezuela, ha reflejado que las plazas de trabajo 
formal se hayan reducido y que el informal crezca donde se ha llegado a estimar que las ciudades que tienen mayor impacto en el mercado laboral son Quito y Guayaquil, siendo Quito, la que tiene mayor índice de desempleo. Para Palacios et al. (2019) otra causa es la reducción de los puestos de trabajo en el sector público lo cual es una decisión producto de la crisis económica que se vive en el Ecuador.

Una realidad que empeora en el área rural, donde solo el 20\% de quienes forman parte de este grupo poblacional tienen un empleo adecuado (Primicias, 2021). Es importante la implementación de políticas públicas, de la ejecución de alianzas público-privadas, debido a que claramente son las empresas las que propician el crecimiento económico, pero es el gobierno el que les da la seguridad a las empresas para que se desempeñen en un buen ambiente social y financiero con todas las garantías para las empresas y sus trabajadores. Además, es necesario implementar medidas que puedan mejorar la competitividad nacional en el corto y mediano plazo, traer prosperidad a las áreas rurales, crear empleos para mujeres y jóvenes, reducir la brecha digital y aumentar el acceso a los mercados financieros (Esquivel, 2020).

\section{Metodología}

La metodología empleada en la presente investigación es de tipo descriptiva. De acuerdo con Aguirre y Jaramillo (2015) la metodología descriptiva tal y como su nombre lo indica se encargan de describir un fenómeno sin que exista intervención del investigador, de esta manera, se procedió a recabar, identificar y analizar antecedentes generales del desempleo en el Ecuador, datos y estadísticas correspondientes al periodo analizado, 2010-2021. A partir de la aplicación de los métodos de investigación inductivo y deductivo, que parten de lo general a lo particular y de lo particular hacia interpretaciones generales, se interpreta racional y objetivamente los datos obtenidos y el impacto de las cifras del desempleo en la población ecuatoriana.

El fundamento teórico de la investigación encuentra sustento en la investigación bibliográfica, el cual, consistente con lo mencionado por Gómez et al., (2014) que indica que la recolección de la información bibliográfica constituye en una de las etapas de mayor importancia debido a que debe garantizar la obtención de la información de mayor relevancia en el ámbito de la investigación. Es así como una vez definido el problema a investigar, fueron consultadas diversas bases de investigación como las siguientes: EBSCO, Redalyc, Scielo, Lantindex, DOAJ. Además, se recurrió a informes de organizaciones e instituciones nacionales e internacionales como: Instituto Ecuatoriano de Estadísticas y Censos (INEC), Banco Central del Ecuador (BCE), Ministerio de Economía y Finanzas del Ecuador, Ministerio del Trabajo, Comisión Económica para América Latina y el Caribe (CEPAL), Organización Internacional del Trabajo (OIT), entre otras.

También se utilizó estudios referenciales al tema para analizar características y condiciones de vida que se han suscitado a lo largo del tiempo en la población ecuatoriana. Para la continuidad del análisis descriptivo se extrajo la información con el propósito de compararla con la nueva evidencia y obtener importantes datos para su interpretación y contextualización con la realidad que se vive en el presente. 


\section{Resultados}

A continuación, se comparten los resultados de la investigación descriptiva histórica del crecimiento de la tasa de desempleo en el Ecuador, se tomó como referencia los años 2010 al 2021. Siendo el mes de mayo del año 2021 el de mayor incremento del desempleo obteniéndose un $6.3 \%$ a nivel nacional (ver figura 1).

Figura 1. Evolución del desempleo en el ecuador: 2010-2021

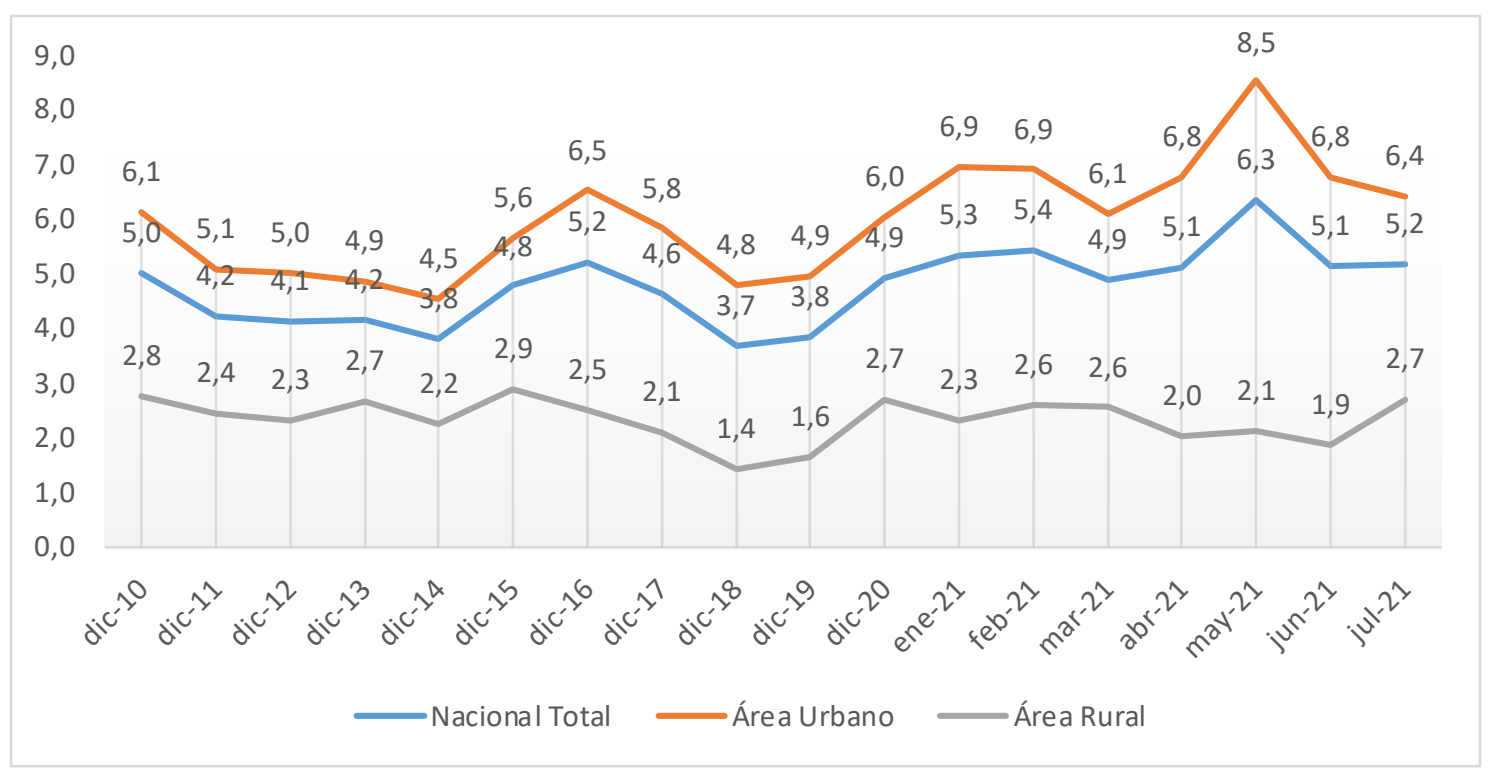

Fuente: INEC

Según datos del INEC el desempleo en el Ecuador desde 2010-2014, refleja tendencia decreciente a partir del 2014. Históricamente se puede observar que existen fluctuaciones en cuanto a la evolución del desempleo que son consecuencia de los factores externos a la economía ecuatoriana, entre estos factores se pueden numerar el desequilibrio experimentado por los precios en los bienes de exportación, el precio de petróleo en los mercados internacionales, entre otros (Castañeda y García, 2019). Son acontecimientos que influyen en el aumento del desempleo para el 2016 llegando a un pico del 6,5\%. En este año Se evidencian las consecuencias de la recesión pues las cifras de desempleo aumentan por la desaceleración de distintos sectores económicos.

El aumento de impuestos y la apreciación del dólar a nivel mundial han provocado menos demanda interna y menor competitividad a exportaciones nacionales en general. Los daños causados por el sismo y la caída del precio del petróleo afectaron los ingresos fiscales del Gobierno, también el flujo de recursos, el gasto público y las perspectivas de crecimiento (Carreño, 2016). Analizando las estadísticas de los últimos 10 años y los primeros meses del 2021 se observa una tendencia creciente del desempleo, siendo preciso incluir en el análisis las consecuencias experimentadas en el ámbito económico y social de la crisis sanitaria provocada por el COVID-19, donde muchas empresas cerraron sus actividades o disminuyeron su producción debido a la disminución de las actividades comerciales. Para junio del 2021 alcanzo el 1,9\% en el área rural sin embargo esta tasa sube en $2,7 \%$ para julio del mismo año. 
En el sector urbano se evidencia una tendencia decreciente en el desempleo, alcanzando $6,4 \%$ para julio del 2021. Estos resultados se derivan del estado de excepción (cuyo fin es el de precautelar los derechos de las personas y controlar las circunstancias de inseguridad producto del COVID-19) a raíz de la extensión del mismo hasta el 30 de julio de 2021 que tanto el Ministerio de Trabajo como el Ministerio de Salud, presenten al Servicio Nacional de Gestión de Riesgos y Emergencias (COE) el modelo que sería implementado para que las personas puedan retomar sus actividades productivas, especialmente para aquellas actividades que se vieron restringidas de llevarlas a cabo de forma presencial (Comité de Operaciones de Emergencia Nacional, 2021).

La pandemia afecta en mayor medida estos resultados, incrementando a escala superior este indicador económico, al mismo tiempo la llegada de extranjeros al Ecuador "En diciembre de 2020 alrededor de 415835 ciudadanos venezolanos estaban residiendo en el país” (Organización Internacional para las Migraciones, 2020). Situación que ha provocado el desplazamiento de las personas desempleadas nuevas hacia el subempleo o empleo informal. Se espera que con la finalización del estado de excepción la tasa de desempleo en Ecuador baje.

Figura 2. Desempleo por sexo: 2010-2021.

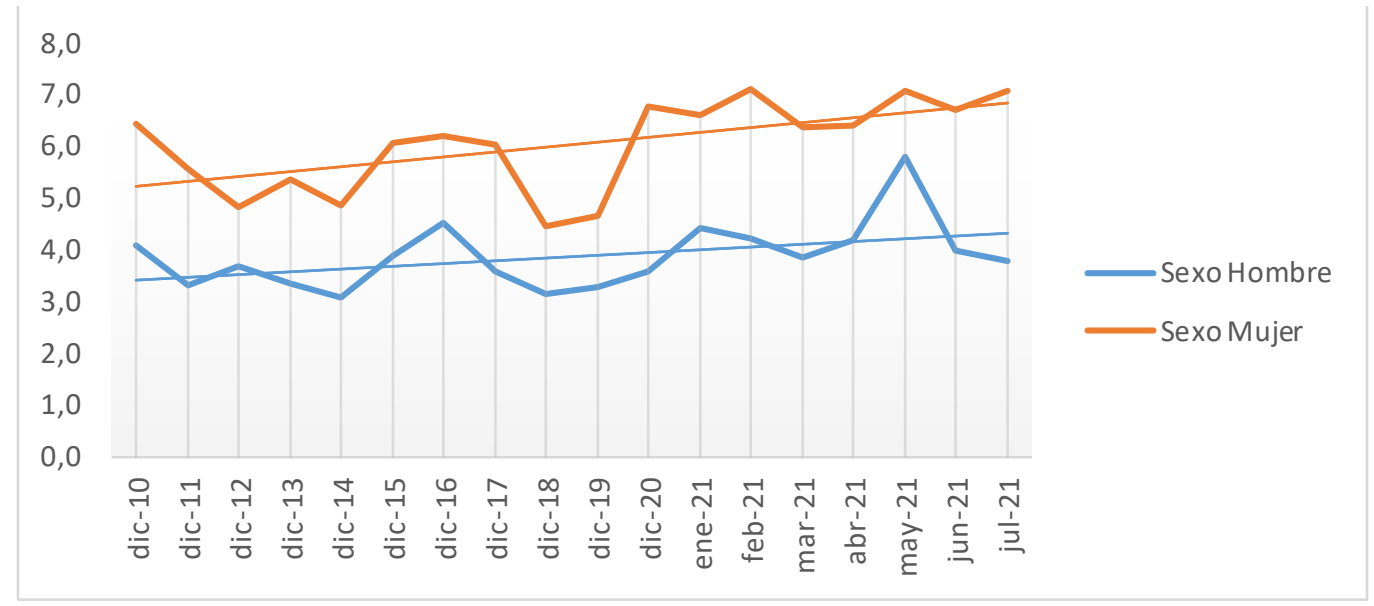

Fuente: INEC 2021

Al comparar la evolución del desempleo desde diciembre del 2010 al momento, se observa una tendencia creciente tanto para los hombres como para las mujeres, sin embargo, el desempleo para las mujeres es mayor que los hombres, se observa una secuencia un tanto similar en lo que corresponde 2010-2019. A partir del 2020 se incrementa la tasa de desempleo en mayor medida para las mujeres, sin embrago para mayo del 2021 la tasa de desempleo de los hombres llega $5,8 \%$ siendo esta la tasa de mayor desempleo en la historia del Ecuador, para junio y julio del presente año este indicador bajo considerablemente, manteniéndose en 3.8\% a comparación de las mujeres donde el desempleo sigue en aumento con 7,1\% para julio del 2021.

De acuerdo con una publicación de Diario El Universo (2021), la mujer profesional se desempeña en muchos campos, debido a que también es madre de familia y dentro de las organizaciones, se analiza de forma errónea el desempeño que tendría, debido a que se sugiere que va a pedir constantemente permiso para atender problemas familiares y actualmente, por el hecho de que muchas mujeres se han sumado a la modalidad teletrabajo se han visto responsables no solo de 
sus actividades profesionales, sino también de las del hogar, por lo que algunos empresarios incluso perciben una disminución de la productividad.

Figura 3. Sectorización del empleo 2010 - 2021.

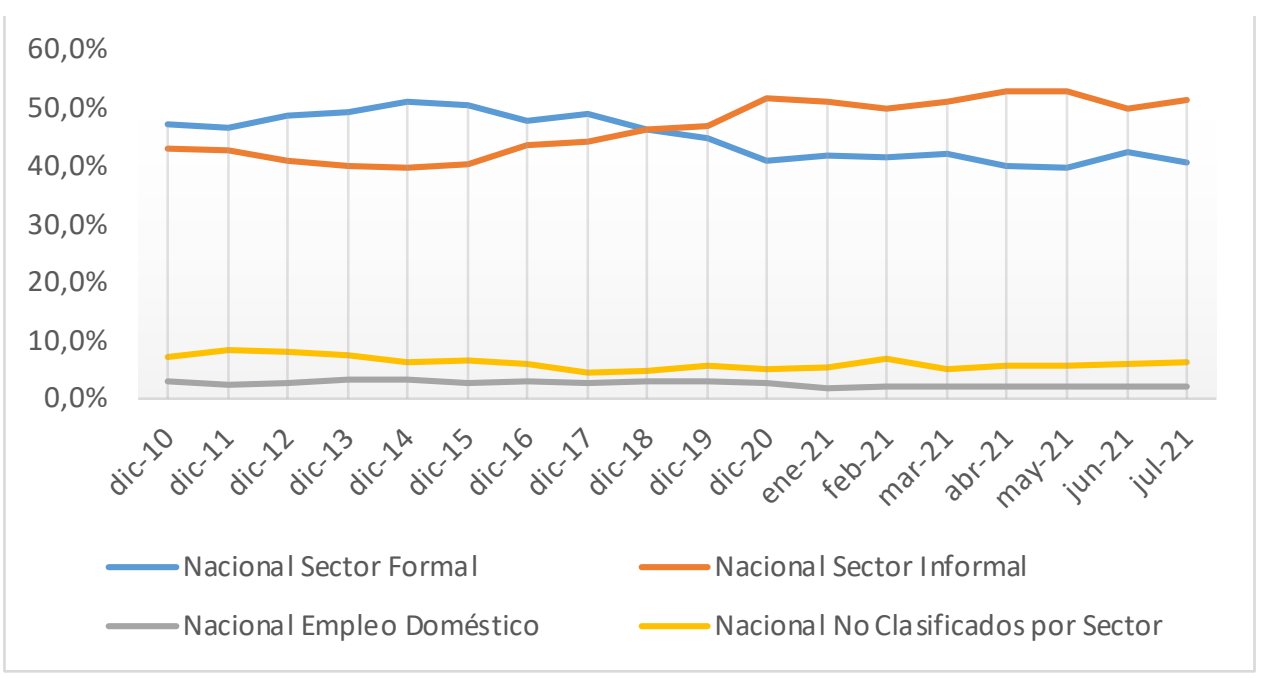

Fuente: INEC 2021

A raíz del aumento en el nivel de desempleo, se evidencia el desplazamiento hacia el sector informal, para finales del 2018 se observa variaciones en el trabajo formal e informal, el empleo en el sector formal comienza a disminuir y el empleo informal empieza a crecer, sin embargo, en el año 2020 se evidencia más en la sociedad ecuatoriana. Para julio del 2021 la tasa de empleo en el sector informal fue $51.2 \%$ y para el sector formal una tasa del $40.6 \%$, el desplazamiento laboral y la economía se ven más afectadas. Mayor cantidad de desempleo está reflejada en la disminución de empleo formal, al dejar de percibir ingresos de manera segura desencadena un efecto de reducción en la economía, viéndose en la necesidad de limitar gastos y buscar nuevo oficio ya sea en el sector formal e informal para salir del desempleo.

Para el 2014 el empleo formal llego a su pico más alto de 50,9\% y el empleo informal para el mismo año llego a su pico más bajo de 39.7\%, a partir del 2014 estos resultados han bajado para el empleo formal y han aumentado para el empleo informal. Sin embargo, estas variables se ven más afectadas con la llegada de la pandemia del COVID-19 en el 2020, manteniéndose en aumentos y disminuciones en los que lleva del 2021.

Ruesga et al., (2020) lleva a cabo una relación con respecto a los bajo niveles educativos y la informalidad del trabajo en los países de desarrollo, es decir, mientras menos accedan a la educación, los individuos se inclinarán por realizar trabajos informales. Contextualizando el incremento del trabajo informal con respecto a la crisis sanitaria experimentada por la pandemia, diario El Telégrafo (2021) llevó a cabo una investigación orientada hacia esa misma problemática, donde los resultados indican que debido a la pandemia, los empresarios se han visto en la situación de despedir trabajadores, los mismos que para poder subsistir y continuar siendo sostén de hogar, se han visto en la obligación de obtener ingresos a partir de sumarse al trabajo informal, situación que se ve incrementada por la presencia de extranjeros que llegan al país y aceptan ejercer este tipo de actividades. 
Figura 4. Desempleo, total (\% de la población activa total) (estimación modelado OIT) Ecuador, Colombia, Perú, Venezuela.

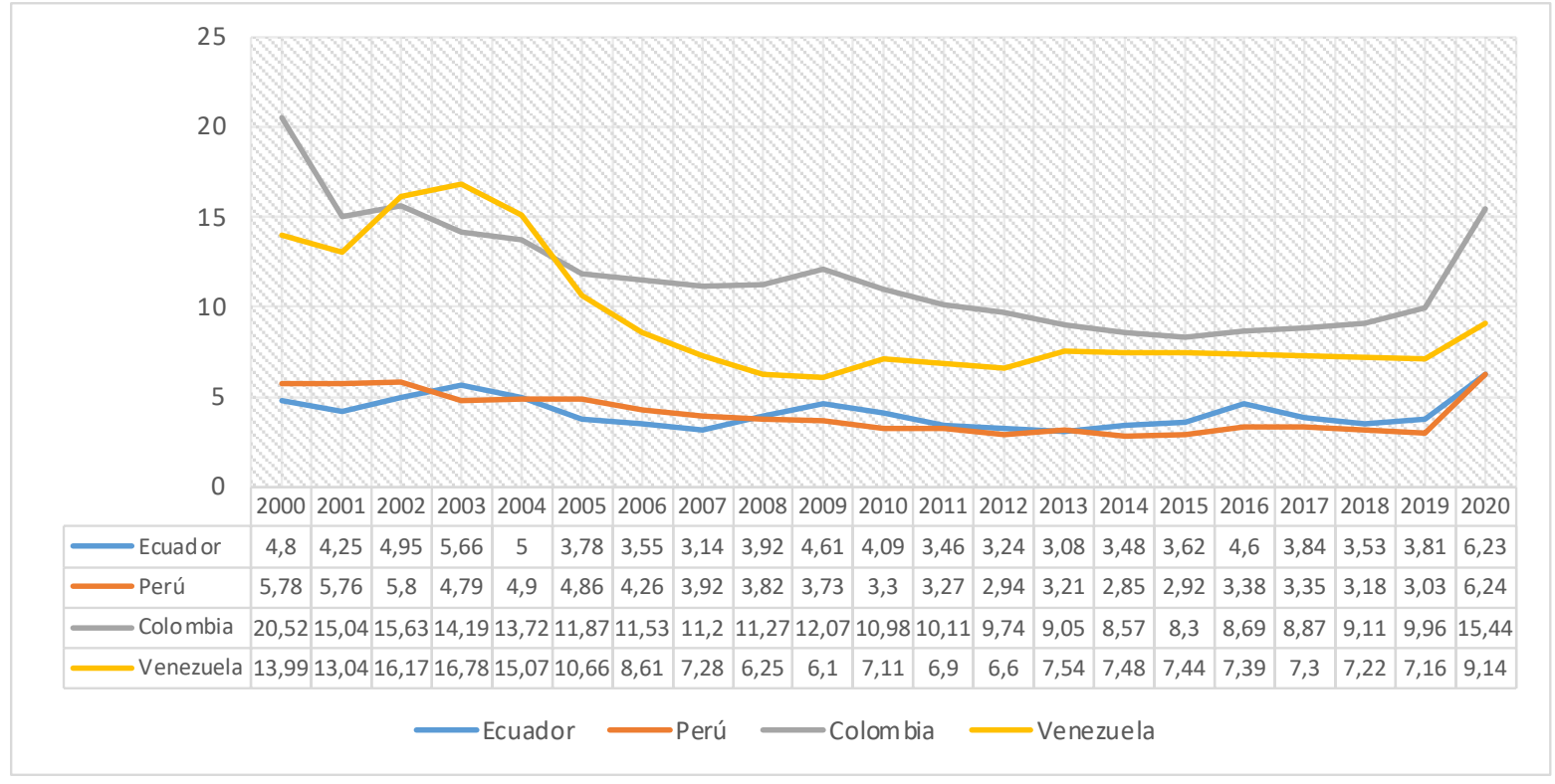

Fuente: BANCO MUNDIAL 2021

El desempleo en Ecuador al igual que Perú, Colombia y Venezuela reflejan tendencias decrecientes desde 2010 hasta el 2020, con la llegada de la pandemia de la COVID-19 se incrementó significativamente la tasa de desempleo en estos países y a nivel mundial, sin embargo Ecuador al igual que Perú se han mantenido en tasas de desempleo semejantes, a comparación de Colombia y Venezuela, estos dos países en lo que corresponde al desempleo desde el 2010 al 2020 siguen estando por encima de Ecuador y Perú. Estas situaciones provocan llegada ciudadanos de esas naciones hacia países como Ecuador y Perú buscando mejorar su calidad de vida (Ramírez y Useche, 2019). La migración en el Ecuador se ha convertido en un fenómeno de gran impacto económico y social, incluso se ha llegado a asociarla con el incremento de la delincuencia y asociaciones ilegales, además de las altas tasas de desempleo en el territorio nacional (Herrera, 2020).

Figura 5. Caracterización del desempleo Nacional.

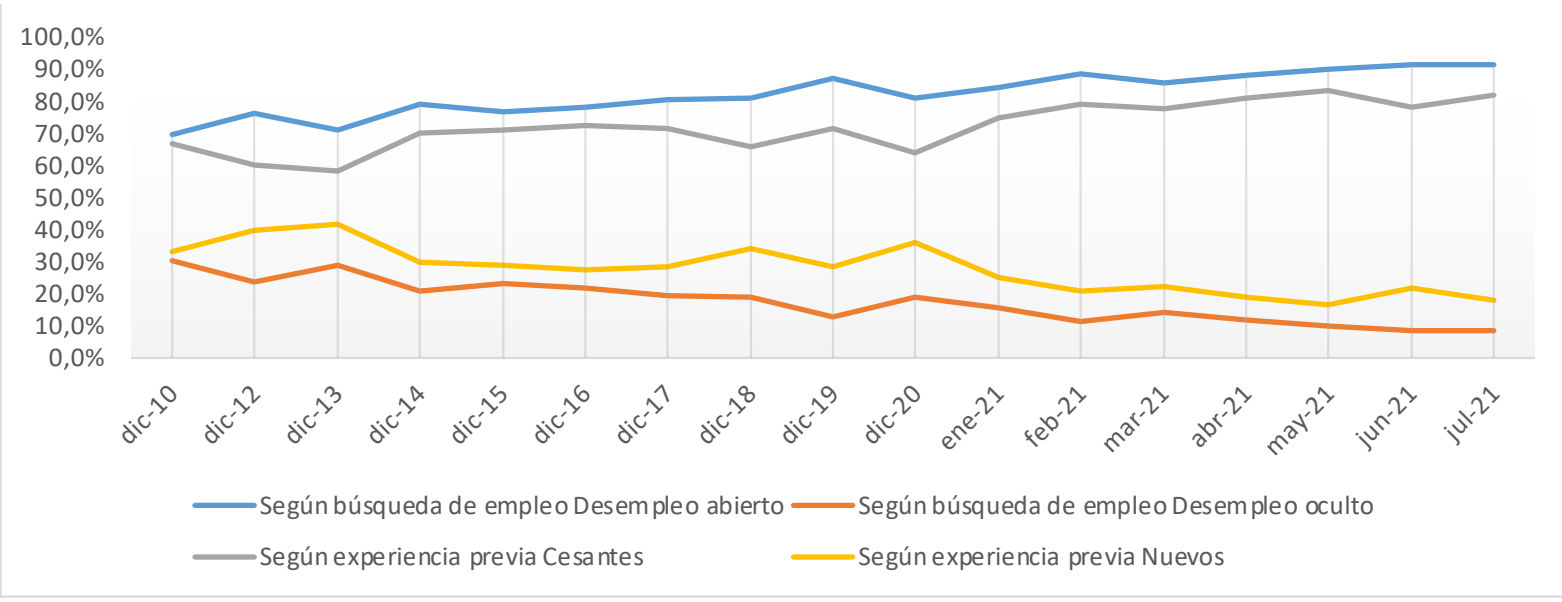

Fuente: INEC 2021 
De acuerdo a los datos de la ENEMDU 2021 se evidencia tendencias crecientes en el desempleo abierto y cesante entre diciembre del 2010 y julio del 2021, se evidencia a nivel nacional que la incidencia de personas que están sin trabajo, buscó empleo en la semana pasada o hicieron gestiones para poner un negocio fue del 21,8\% siendo la tasa más alta en comparación a las otras variables del desempleo, en la cesantía su aumento fue del 15\%. Para el mismo periodo, el desempleo oculto muestra tendencia decreciente del 21,8\% y el desempleo nuevo decreció en un $13 \%$ respectivamente.

Figura 6. Desempleo urbano - rural, principales ciudades: enero - marzo 2021.

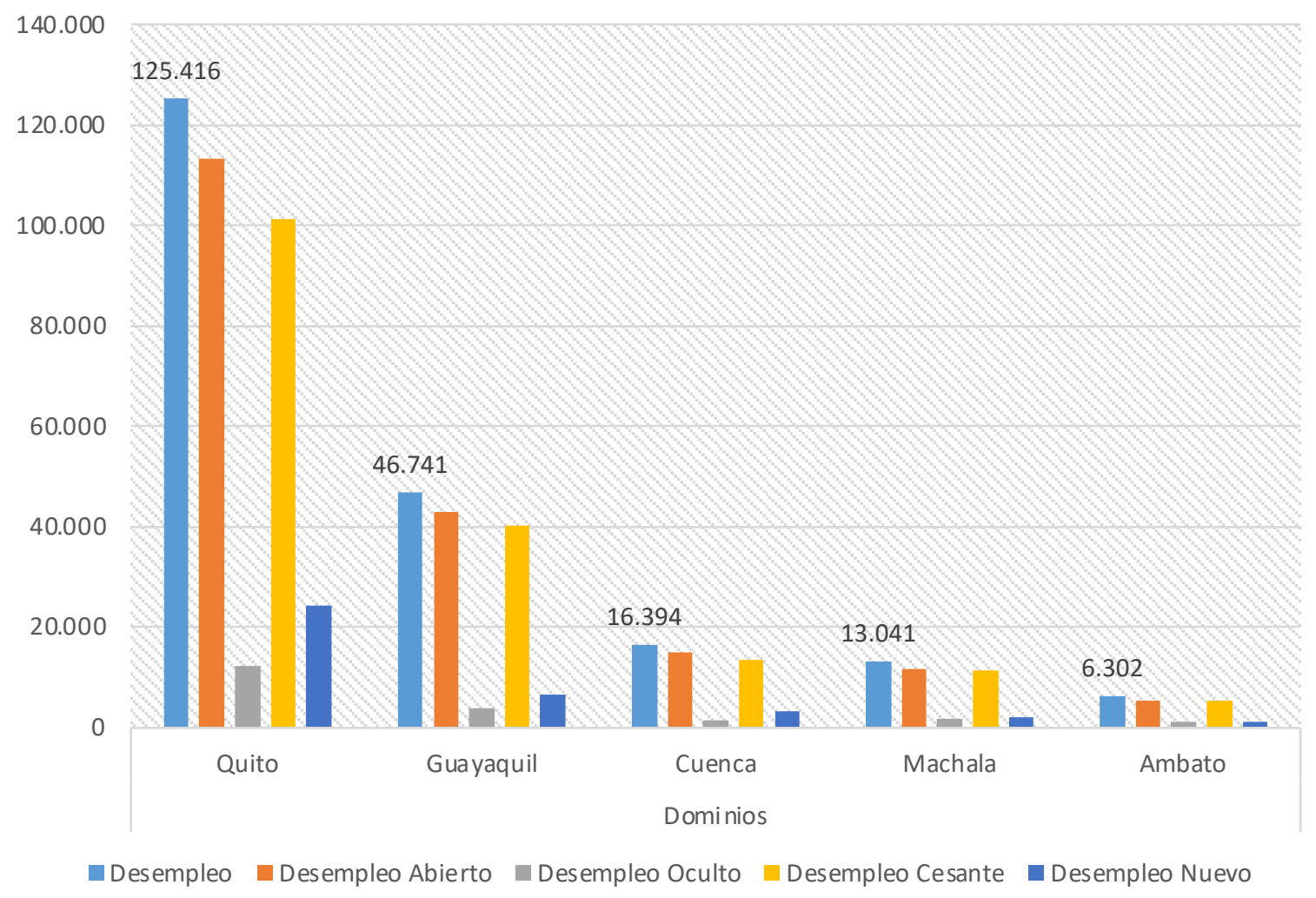

Fuente: INEC 2021

En el primer trimestre del 2021 las ciudades con más personas desempleados son Quito y Guayaquil, tanto en el desempleo abierto como en el desempleo cesante, se encuentra que de enero a marzo del 2021 Quito cuenta con 125.416 personas que están en condición para laborar sin embargo no tienen la disposición por la falta de vacantes disponibles. La ciudad de Ambato en cambio reflejó la menor cantidad de desempleado con un total de 6.302 personas, resultados favorables en su primer trimestre del 2021.

\section{Discusión}

La lucha contra el desempleo es constante en todos los países del mundo debido a que como lo menciona Quiroa (2019) este impacto puede evidenciarse al momento de llevar a cabo los análisis de las tasas de crecimiento del desempleo y la influencia que tiene en el desplazamiento hacia la realización del trabajo informal. Rodríguez (2018) que, en ámbito de Latinoamérica, el 
desempleo se ha visto impactado principalmente por las fluctuaciones del tipo de cambio real, provocando que sus productos se vuelvan muy costosos, y generando así, el ingreso masivo de productos extranjeros a precios más bajos que los nacionales. Todo esto, ha generado una disminución de las ventas nacionales y una baja en la demanda agravando aún más las tasas de desempleo.

Ecuador con el cambio de moneda logra disminuir los niveles del desempleo y pobreza considerablemente para el año 2010, mismo que se toma como punto de referencia por su favorable indicador y para análisis posteriores. A partir de los hallazgos encontrados se establece que existe relación en el incremento del desempleo en las mujeres y los jóvenes del país, se refleja una tendencia decreciente del desempleo hasta el periodo 2014, sin embargo para años posteriores se refleja un aumento en la tasa del desempleo de distintos sectores, al comparar la evolución del desempleo desde diciembre del 2010, se observa una tendencia creciente tanto para los hombres como para las mujeres, sin embargo el desempleo para las mujeres es mayor que los hombres.

Estos resultados guardan relación con lo que sostienen (Rodríguez, 2018) quienes señalan que las mujeres y los jóvenes son los más afectados, ya que las mujeres registraron un incremento de 2 puntos porcentuales (9,8\%) con respecto al 2015 (7,8\%), y el desempleo en los jóvenes incremento más de 3p.p. (18,3\%) con respecto al 2015 (15,1\%). Por otra parte, León et al., (2020) en los resultados de su investigación, indica que la implementación de programas para la creación de empleo como las obras en construcción, proyecto de innovación hidráulica, construcción de vías, entre otras, fueron parte de los mecanismos para el descenso del indicador de pobreza y desempleo para el año 2018 llegando a ubicarse en una tasa del 3,7\%.

En comparación con otros estudios como El desempleo en el Ecuador: causas y consecuencias realizado por Sumba et al., (2020), las similitudes en los resultados son claras, en temas como la migración, la pandemia del COVID-19, la caída del precio del petróleo, etc. Son algunos de los factores determinantes en el análisis de desempleo además de mostrar su impacto tanto en el ámbito económico como social.

En lo que respecta al desempleo en el área rural en los últimos 10 años y en los primeros siete meses del 2021 refleja una tendencia creciente siendo el año 2020 con mayor desempleo rural. Para junio del 2021 alcanzo el 1,9\% en el área rural sin embargo esta tasa sube en $2,7 \%$ para julio del mismo año. Entre los efectos del desempleo en países como Ecuador al igual que Perú, Colombia y Venezuela reflejan tendencias decrecientes desde 2010 hasta el 2020, con la llegada de la pandemia de la covid-19 se incrementó significativamente la tasa de desempleo en estos países y a nivel mundial.

\section{Conclusión}

Se llega a la conclusión que el desempleo en el Ecuador se ha incrementado, la economía ecuatoriana ha experimentado un gran impacto con la llegada de la pandemia y el confinamiento obligatorio para disminuir los contagios del virus. No se ha logrado grandes cambios y se evidencia en el desplazamiento del desempleo hacia el empleo informal. Con la llegada de la pandemia (COVID-19), se encontró que el desempleo rural no se afectó a niveles tan elevados 
como el desempleo urbano, sin embargo, para julio del 2021 la tasa de desempleo en el área rural es la más alta aumentando $0,8 \%$ con respecto al mes anterior.

Se evidencian resultados consistentes a estudios similares como el desplazamiento de la mano de obra hacia el sector informal, sin embargo, para el nuevo estudio que busca analizar el desempleo hasta el 2021, en este caso se analizara nuevas variables que intervienen en el incremento del desempleo tales como: la pandemia del COVID-19 y la llegada de extranjeros al país en mayor cantidad.

De la misma forma el desempleo urbano es el más afectado debido a que las empresas se ven obligadas a despedir personal por falta de rentabilidad, también se refleja mayor desempleo en las mujeres ya sea por la preferencia de los directivos en personal masculino debido a que no se involucran tanto como las mujeres en cuestiones del hogar. Sin embargo, para el nuevo estudio se evidencia tasas más altas, ubicándose para julio del 2021 en 7,1\% para las mujeres y 3,8\% para los hombres.

Lo mismo sucede con los resultados del desempleo en las principales ciudades del país, se evidencia resultados semejantes en comparación con estudios anteriores, las ciudades con mayor gente desempleada en el primer trimestre del 2021 son Quito con 124416 y Guayaquil 46.741, Al bajar el gasto público en 2016 se evidencio mayor crecimiento del desempleo, si bien los ajustes que se realizaron en el gasto público del 2014 hasta 2016 no fueron favorables según los resultados obtenidos. Se espera que la economía vuelva a reactivarse cuando termine el estado de excepción, a través del cual se busca precautelar los derechos de las personas en el Ecuador.

\section{Referencias}

Aguirre, J., \& Jaramillo, L. (2015). El papel de la descripción en la investigación cualitativa. Cinta moebio, 53, 175-189. http://doi.org/10.4067/S0717-554X2015000200006

Briales, Á. (2017). Emprendeudores fracasados: individualización neoliberal en los discursos sobre el desempleo. RECERCA, 1(20), 79-104. https://www.e-revistes.uji.es/index.php/recerca/article/ view/2188/2082

Campoverde, A., Ortíz, C., \& Sánchez, V. (2016). Relación entre la inflación y el desempleo: una aplicación de la curva de Phillips para Ecuador, Latinoamérica y el Mundo. Revista Económica, 22-34. https:// revistas.unl.edu.ec/index.php/economica/article/view/200

Carreño, S. (2016, julio 25). Al ‘boom’ económico de Ecuador le llegó la recesión. El Tiempo. https://www. eltiempo.com/archivo/documento/CMS-16654045

Castañeda, G., \& García, K. (2019). Análisis del incremento del desempleo en el Ecuador, período 2014 2018. Revista Observatorio de la Economía Latinoamericana. https://www.eumed.net/rev/oel/2019/11/ incremento-desempleo-ecuador.html

Chen, C. (1990). Economía laboral contemporánea Teorías y políticas. Academia Nacional de Ciencias Económicas.

Comité de Operaciones de Emergencia Nacional. (2021, julio 9). Resoluciones. COE Nacional, viernes 09 de julio de 2021. COE. https://cutt.ly/YYKdTbR

Esquivel, M. (2020, junio 22). La pandemia del desempleo. INCAE Business School. https://www.incae.edu/ es/blog/2020/o6/22/la-pandemia-del-desempleo.html 
Gómez, E., Fernando, D., Aponte, G., \& Betancourt, L. (2014). Metodología para la revisión bibliográfica y la gestión de información de temas científicos, a través de su estructuración y sistematización. Dyna, 81(184), 158-163. https://doi.org/10.15446/dyna.v81n184.37066

Herrea, D. (2020, agosto 13). Los refugiados y migrantes en Ecuador enfrentan riesgos crecientes ante una reducción de las protecciones. Open Global Rights. https://cutt.ly/PYKdNc7

León, L., Vargas, K., \& Zúñiga, F. (2020). Ecuador: Incidencia de la pobreza en el producto interno bruto y desempleo 2008-2018. Revista de Investigación Enlace Universitario, 19(2), 1-13. http://doi.org/10.33789/ enlace.19.2.69

López, F. (2020). Dinámica de los flujos de entrada y salida del desempleo en Chile 1996-2016. Revista de Análisis Económico, 33(2). http://dx.doi.org/10.4067/S0718-88702018000200003

Monitoreo Internacional para las Migraciones. (2020). Monitoreo de Flujo de Población Venezolana. OIM.

Organización Internacional del Trabajo. (2021). Crecimiento económico con alto coeficiente de empleo. OIT. https://cutt.ly/AYKd9g9

Palacios, N., Gómez, X., \& García, K. (2019). Análisis de variación de la tasa de desempleo y su incidencia en las condiciones de vida del Ecuador. Periodo 2013-2017. Revista Observatorio de la Economía Latinoamericana.

Polany, K. (1994). El sustento del hombre. Mondadori.

Primicias. (2021, septiembre). Apenas dos de cada 10 trabajadores del campo tienen un empleo adecuado. Primicias Economía https://cutt.ly/IYKfuFL

Quiroa, M. (2019, agosto 8). El desempleo, un problema actual. Economipedia. https://economipedia.com/ actual/el-desempleo-un-problema-actual.html

Ramírez, Y., \& Useche, E. (2019). Geopolíticas migratorias, inserción laboral y xenofobia: migrantes venezolanos en Ecuador. En C. Blouin. Después de la Llegada. Realidades de la migración venezolana. Themis-PUCP

Rodríguez, E. (2018). Análisis del desempleo en Ecuador en el periodo 2010-2017. Un enfoque econométrico. (Tesis) Universidad del Azuay. http://dspace.uazuay.edu.ec/handle/datos/7724

Ruesga, S., Pérez, L., \& Delgado, J. (2020). Sector informal en Ecuador: Perspectiva desde el escenario econométrico. Espacios, 41(14), 1-14.

Smith, A. (1958). Investigación sobre la naturaleza y causas de la riqueza de las naciones. México.

Sumba, R., Saltos, G., Rodríguez, C., \& Tumbaco, Z. (2020). El desempleo en el ecuador: causas y consecuencias. Polo del Conocimiento, 5(10), 774-797.

Torres, J., \& Montero, A. (2005). Trabajo, empleo y desempleo en la teoría económica: la nueva ortodoxia. Principios, 3(1), 5-34.

Vallejo, L. (2010). El desempleo y la pobreza. Revista Apuntes del CENES, 29(49), 7-8. https://revistas.uptc. edu.co/index.php/cenes/article/view/53

\section{AUTORES}

Josthin Ramirez. Egresado de Economía de la Universidad Técnica de Machala, Ecuador.

Jonh Campuzano. Master en administración de empresas. Economista y Coordinador del grupo de investigación en Desarrollo Económico y Territorial. 